\title{
The Effect of Correlated Noise in a Gompertz Tumor Growth Model
}

\author{
Anita Behera \\ School of Biomedical Sciences, \\ Queen's University of Belfast, BT9 7AB, United Kingdom \\ S. Francesca C. O'Rourke \\ School of Mathematics \& Physics \\ Queen's University of Belfast, BT7 1NN, United Kingdom
}

Received on 3 April, 2008

\begin{abstract}
We study the effect of noise in an avascular tumor growth model. The growth mechanism we consider is the Gompertz model. The steady state probability distributions and average population of tumor cells are analyzed within the Fokker-Planck formalism to investigate the importance of additive and multiplicative noise. We consider the effect of correlation on tumor growth for both the case of nonzero and zero correlation time. It is observed that the Gompertz model, driven by correlated noise exhibits a stochastic resonance and phase transition. This behaviour is attributed to multiplicative noise. In the case of nonzero correlation time, it is found that the correlation strength and correlation time have opposite effects on the steady state probability distribution. The Gompertz model simulations are also shown to be in qualitative agreement with another similiar non-bistable system, the logistic model.
\end{abstract}

Keywords: Fluctuation phenomena; Random processes; Noise; Brownian motion

\section{INTRODUCTION}

Much of the attention in the recent years has been directed towards nonlinear physics and its application to uncover biological complexities. Studies have confirmed the role of noise in the nonlinear stochastic systems [1]. In recent years the Fokker-Planck equation has become one of the important approaches in the studies of nonlinear dynamics based on stochastic form $[2,3]$.

Tumor growth is a complex process. It has been a challenge for many years to search for a suitable growth law of tumors. Mathematical models based on mathematical equations such as the Gompertzian, logistic and exponential equations are used as a basic tool for describing avascular tumor growth [4-6]. It has been realized that tumor growth is governed by environmental fluctuations [7]. It has also been shown that quite often there are discrepancies between theoretical predictions and clinical data due to more or less intense environmental fluctuations [8]. Ferreira et al [6] analyzed the effect of distinct chemotheraputic strategies for the growth of avascular tumors. This study confirmed that an environment like chemotherapy affects tumor growth behaviour and may lead to morphological transitions under certain conditions.

Stochastic modelling using the Fokker-Planck equation has been recently applied to the logistic equation to study the steady state properties of avascular tumor growth driven by Gaussian correlated additive and multiplicative white noise by [9]. This work has been extended to consider the nonzero correlation time between additive and multiplicative noise by [10]. However among avascular tumor growth power laws the Gompertz model has been the most broadly and successfully applied to fit the experimental data [11-13]. Other successful applications in the literature of the Gompertz equation to cancer cell growth include [14-16]. These models are however deterministic models. In this article we wish to consider the
Gompertz model from a stochastic viewpoint by examining the steady state properties of Gompertzian tumor cell growth driven by correlated noise. The Gompertz model has not been analyzed in the literature before in this context. In addition we extend our study to consider the nonzero correlation time for the Gompertz model.

The plan of this paper is as follows. Calculational details outlining the theoretical formulation of the Fokker-Planck equation applied to avascular tumor growth laws are presented in Sec.II. Sec.III presents the results for the steady state properties and average cell populations of the Gompertz model driven by cross-correlated additive and multiplicative noise for the case of zero correlation time between noises. In Sec.IV we consider nonzero correlation time and present the results for the steady state properties of the Gompertz model driven by cross-correlated noise for the case of nonzero correlation time. We use our model to simulate the behaviour of the logistic model of [9], [19], and [10] and compare with the results obtained from the Gompertz model.

Finally in Sec.V we discuss our conclusions.

\section{THEORETICAL FORMULATION BASED ON THE FOKKER-PLANCK EQUATION}

Avascular tumor growth laws may be described by a single deterministic differential equation,

$$
\frac{d x}{d t}=f(x) \quad \text { with } x(0)=x_{0},
$$

where $f(x)$ describes the tumor cell growth dynamics and $x_{0}$ is the initial tumor size.

Typically the modelling assumptions that Eq.(1) are based on, are $(i)$ the tumor only contains one cell type, (ii) is spatially independent, (iii) does not explicitly mention nutrients, 
growth factors or host vasculature, (iv) tumor volume is proportional to $x(t)$, the number of tumor cells at time $t$.

The Gompertz law may be modelled by taking

$$
f(x)=-b x[\log (x / \kappa)],
$$

where $x$ is the tumor cell number, $b$ the cell decay rate, $\kappa$ is carrying capacity, where $\kappa=\frac{a}{b}$, and $a$ is the cell growth rate. Gompertz growth is a result of two classes of competitive processes, the first process simulates growth and the second phase constrains growth at the saturation stage. Another alternative growth law which behaves similarly to the Gompertz model is the logistic model. The logistic model is governed by the equation where,

$$
f(x)=a x-b x^{2} .
$$

The solution of this equation belongs to same class of sigmoidal functions as the Gompertz model. It models growth exponentially in the early stages but eventually saturates due to the quadratic term in the above equation.

Eq. (1) can be generalized to consider stochastic effects due to external factors such as temperature, drugs, radiotherapy etc, by introducing, $(i)$ Gaussian multiplicative noise to represent the effect of the treatment by altering the tumor cell dynamics (ii) a negative additive Gaussian noise which may represent fluctuations due to the treatment resulting in cell death. Here we have implicitly assumed both the multiplicative and additive noise are correlated since they have a common origin. Modelling this stochastic behaviour yields,

$$
\frac{d x}{d t}=f(x)+x \varepsilon(t)-\Gamma(t),
$$

where $\varepsilon(t)$ and $\Gamma(t)$ are Gaussian multiplicative and additive noises respectively with the following properties:

$$
\begin{gathered}
\langle\varepsilon(t)\rangle=\langle\Gamma(t)\rangle=0, \\
\left\langle\varepsilon(t) \varepsilon\left(t^{\prime}\right)\right\rangle=2 D \delta\left(t-t^{\prime}\right), \\
\left\langle\Gamma(t) \Gamma\left(t^{\prime}\right)\right\rangle=2 \alpha \delta\left(t-t^{\prime}\right), \\
\left\langle\varepsilon(t) \Gamma\left(t^{\prime}\right)\right\rangle=2 \lambda \sqrt{D \alpha} \delta\left(t-t^{\prime}\right),
\end{gathered}
$$

where the parameters $D$ and $\alpha$ represent intensity of the multiplicative and additive noises respectively, and $\lambda$ denotes the strength of correlation between $\varepsilon(t)$ and $\Gamma(t)$ with $0 \leq \lambda<1$. Eq.(8) describes zero correlation time between additive and multiplicative noises. To study the effect of non-zero correlation time $\tau$, the statistical property $\left\langle\varepsilon(t) \Gamma\left(t^{\prime}\right)\right\rangle$ is given by the more generalized form,

$$
\left\langle\varepsilon(t) \Gamma\left(t^{\prime}\right)\right\rangle=\left\langle\Gamma(t) \varepsilon\left(t^{\prime}\right)\right\rangle=\frac{\lambda \sqrt{\alpha D}}{\tau} \exp \left[-\frac{\left|t-t^{\prime}\right|}{\tau}\right] .
$$

Eq.(9) reduces to Eq.(8) when $\tau \rightarrow 0$. According to the Langevin Eq.(4), we can derive the Fokker-Planck Equation for the positive values of $x$ [17], which is given by,

$$
\frac{\partial p(x, t)}{\partial t}=-\frac{\partial}{\partial x}[A(x) p(x, t)]+\frac{\partial^{2}}{\partial x^{2}}[B(x) p(x, t)],
$$

where $p(x, t)$ is the probability distribution function, $A(x)$ and $B(x)$ are the drift and diffusion coefficients respectively defined as

$$
\begin{aligned}
& A(x)=f(x)+D x-\lambda \sqrt{D \alpha}, \\
& B(x)=D x^{2}-2 \lambda \sqrt{D \alpha} x+\alpha .
\end{aligned}
$$

According to the reflecting boundary condition, the steadystate probability distribution function (SPDF) of Eq.(10) can be obtained from [17], [18]. This is given by,

$$
p_{s t}(x)=\frac{N}{B(x)} \exp \left[\int^{x} \frac{A\left(x^{\prime}\right) d x^{\prime}}{B\left(x^{\prime}\right)}\right],
$$

where $N$ is the normalisation constant. Eq.(13) may be solved numerically to obtain the steady state probability distribution $p_{s t}(x)$ and average cell population $\langle x\rangle$ for the Gompertz and logistic models.

\section{EFFECT OF ZERO CORRELATION TIME ON AVASCULAR TUMOR GROWTH LAWS}

In this section we study the properties of tumor cell growth using the Gompertz law in the presence of cross correlated additive and multiplicative noises for the case of zero correlation time between noises. Although of course strictly speaking the correlation time is never actually equal to zero, we adopt this approach as a first approximation in considering the biological system driven by noise. This corresponds to the case where we deal only with correlated Gaussian white noise. This is valid biologically when the time scale for the correlation is much shorter than the time scale for the relaxation of the driven process.

In our tumor growth modelling, we assume that the stochastic characteristics arise from both external and internal factors. We further assume that external factors represent fluctuations due to some external factors such as treatments, i.e, chemotherapy or radiotherapy. These treatments affect the tumor's net growth rate generating multiplicative noise and at the same time they restrain the number of tumor cells which give rise to additive noise $[9,10]$.

\section{A. Gompertzian growth}

The results of our calculations for the steady state probability $p_{s t}(x)$ as a function of cell number $x$ are presented in Figs. $1 \mathrm{a}$ and $1 \mathrm{~b}$. In particular, we have studied the effect of the intensity of correlation strength $\lambda$ between the additive and multiplicative noise intensities on $p_{s t}(x)$. It is evident from the Fig. 1a that with the increase of $\lambda, p_{s t}(x)$ decreases at smaller $x$ values ie, $x \leq 5$. However, the peak of $p_{s t}(x)$ increases with higher values of $\lambda$ and at higher $x$ values, $p_{s t}(x)$ for different $\lambda$, merge with each other and die out. This implies that higher values of $\lambda$ promote cell growth. In Fig. $1 b$ we have studied the effect of negative correlation strength on $p_{s t}(x)$. It is interesting to note that as the value of $\lambda$ decreases, $p_{s t}(x)$ increases 


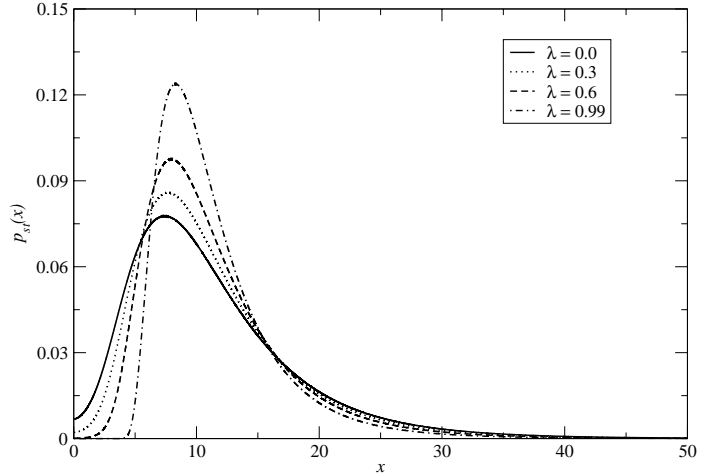

(a)

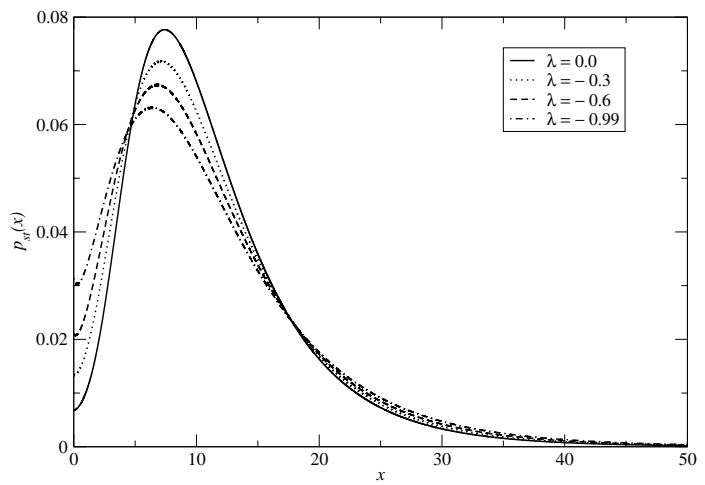

(b)

FIG. 1: (a) Plot of $p_{s t}(x)$ as a function of $x$ for different values of positive correlation parameter $\lambda$. We have used $a=1, b=0.1, D=$ 0.3 and $\alpha=3.0$. (b) Plot of $p_{s t}(x)$ as a function of $x$ for different values of negative correlation parameter $\lambda$. All other parameters are same as (a). Parameter values are in arbitrary units.

at small $x$, and decreases at large $x$. This implies that decreasing $\lambda$ causes the tumor cells to disappear. In other words the distribution of the cell population which was mainly peaked about zero (for smaller values of $\lambda$ ) signifies high extinction rates, and moves towards zero with the decrease of the correlation parameter $\lambda$. Similar behaviour has also been obtained in the case of logistic growth [19]. Comparing Figs. 1a and 1b, we found that a change in sign concerning the correlation between additive and multiplicative noises has both positive and negative effects on tumor growth. Indeed, a negative value of $\lambda$ (which corresponds to a shift to lower values of the peak position of the $\left.p_{s t}(x)\right)$ has a meaning in this context. It corresponds to a change in sign of the correlation between additive and multiplicative noise, which corresponds to a better interpretation of the Eq. (4). In fact, in Eq. (4) the additive and multiplicative noise have opposite effects (when one is positive the other is negative). Therefore, it seems that a positive $\lambda$ indicates a negative feedback between the two noises. On the contrary a negative $\lambda$, or alternatively a change in sign of either of the two noise terms in Eq.(4), may be correct, to simulate positive feedback of two effects in the tumor treatment.

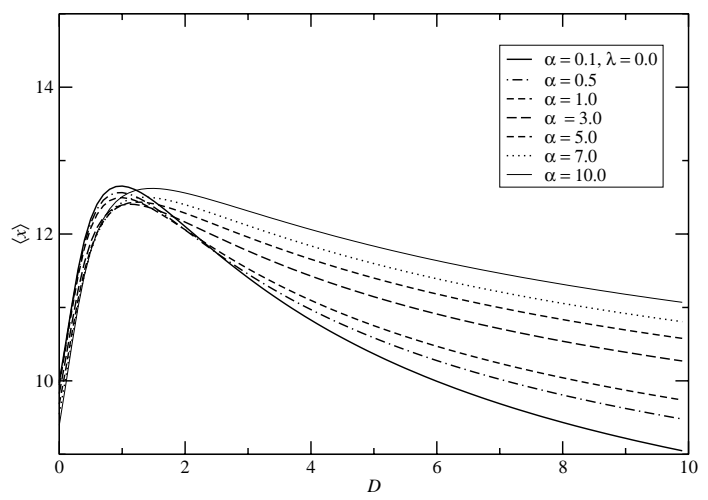

FIG. 2: Plot of $\langle x\rangle$ as a function of $D$ for different values of $\alpha, \lambda=$ 0.0. All other parameter values are the same as Fig. 1.

In Fig. 2, we present our results for average cell population $\langle x\rangle$ as a function of the intensity of multiplicative noise $D$ for different values of additive noise intensity $\alpha$ at a fixed $\lambda=0.0$. This is given by the equation,

$$
\langle x\rangle=\frac{\int x p_{s t}(x) d x}{\int p_{s t}(x) d x} .
$$

We observe from Fig. 2 that $\langle x\rangle$ increases first, and then decreases with the intensity of the multiplicative noise, showing a typical stochastic resonance characteristic. This means that the appropriate intensity of multiplicative noise is suitable for tumor cell growth and extra noise may restrain tumor cell growth. It is evident from Fig. 2 that as $\alpha$ increases the peaks die out and become flat. In Fig. 4, we also observe the same qualitative behaviour for the logistic model.

In Fig. 3a we present the results for $p_{s t}(x)$ as a function of $x$ at various values of $\alpha$. This figure shows that the position of the peaks of the distribution remains unchanged as the intensity of the additive noise increases. However, the height of the peaks decrease as $\alpha$ increases. In Fig. 5a, we also observe the same qualitative behaviour for the logistic model.

We also show in Fig. $3 b$ the average cell population $\langle x\rangle$ as a function of $\alpha$ for different values of multiplicative noise intensity $D$. We observe that $\langle x\rangle$ decreases and then increases showing a negative stochastic resonance-like characteristic at the values of $D$ between 0.3 and 0.9 . This is caused by the amplification of a weak periodic signal by means of noise. The resonances die out with increasing $D$. The critical phase transition occurs at the point of inflection, $\frac{\kappa}{e}$, from the deterministic Eq. (2) due to the sigmoidal nature of the Gompertz model. For comparison we show the results of the logistic model in Fig. 5(b). These results show a similiar trend to the Gompertz model. It may be noted that as the point of inflection, $x=a / 2 b$ in the logistic model, (obtained from the deterministic Eq. (3)), is reached more slowly than in the Gompertz model, the resonances in Fig. 5b are more pronounced than in the Gompertz model in Fig. 3b. These resonances also die out with increasing $D$ as in the Gompertz model. In both models, multiplicative noise induces a phase transition and resonance in tumor growth. This may require further study in or- 


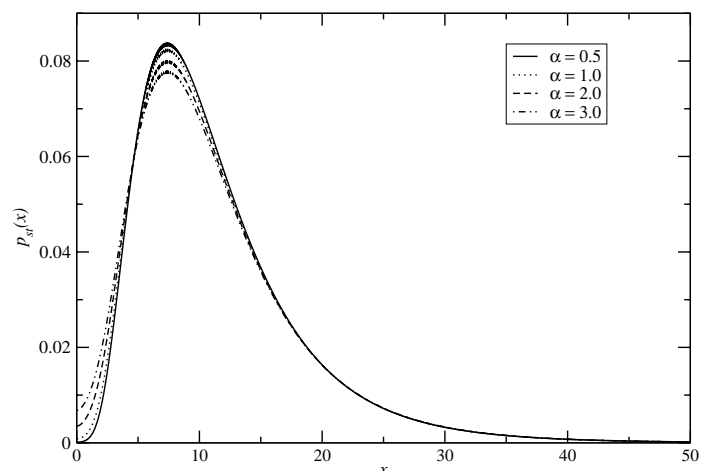

(a)

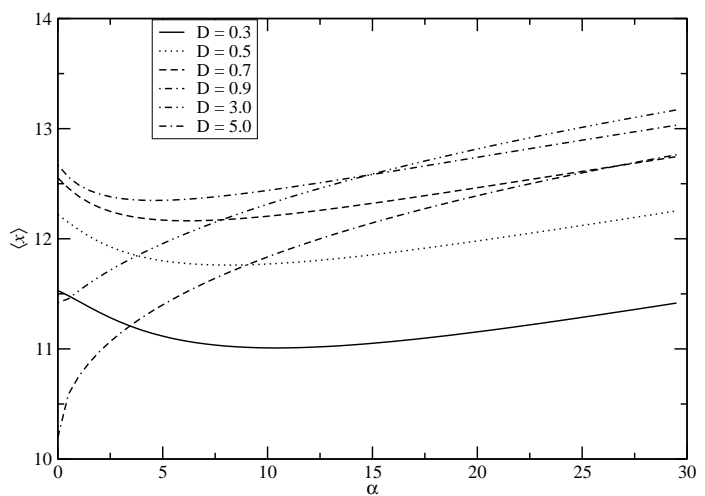

(b)

FIG. 3: (a) Plot of $p_{s t}(x)$ as a function of $x$ for different values of $\alpha$. (b) Plot of $\langle x\rangle$ as a function of $\alpha$ for different values of $D$. All other parameter values of both Figs. $3 \mathrm{a}$ and $3 \mathrm{~b}$ are the same as Fig. 1 .

der to understand the occurance of stochastic resonance-like phenomena and the underlying mechanism(s) in the present non-bistable systems.

\section{EFFECT OF FINITE CORRELATION TIME ON AVASCULAR TUMOR GROWTH MODELS}

In this section we study the stationary properties and average populations of tumor cell growth using the Gompertz and logistic laws in the presence of cross correlated additive and multiplicative noises for the case of nonzero correlated time between noises. This reflects the inclusion of coloured noise into our system and is more appropriate in representing a non-sharp separation of time scales and a greater precision in analysis of the stochastic process. This generalization relaxes the restriction that the correlation time is zero which is the case when considering Gaussian white noise as in Sec. III above.

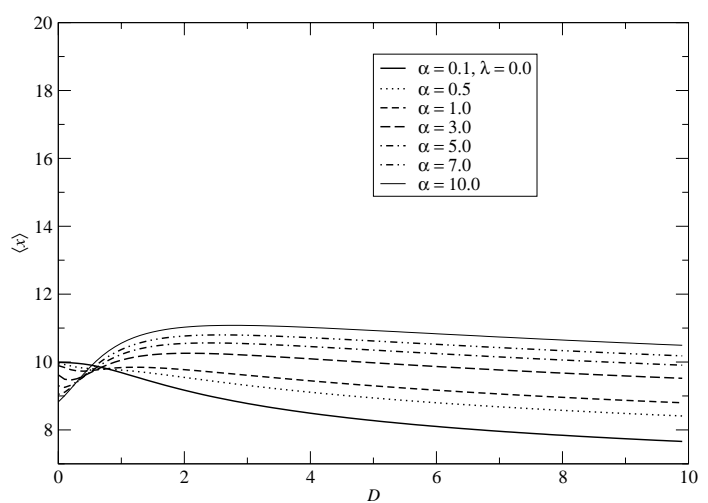

FIG. 4: Plot of $\langle x\rangle$ as a function of $D$ for different values of $\alpha$. All other parameter values are the same as Fig. 1.

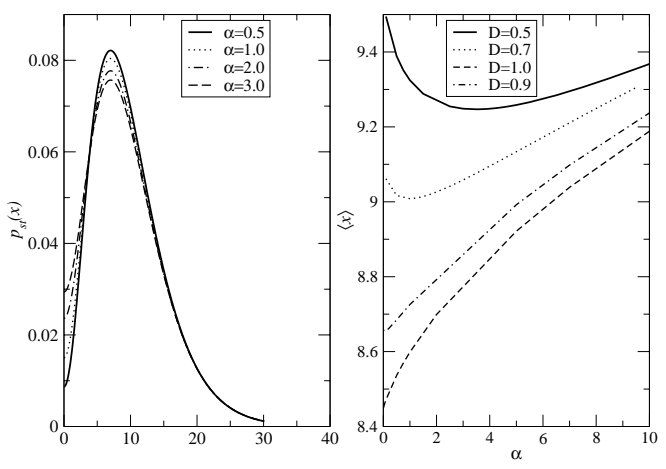

FIG. 5: Plot of $p_{s t}(x)$ as a function of $x$ (left) for different values of $\alpha$ and for fixed values of $D=0.3$. Plot of $\langle x\rangle$ as a function of $\alpha$ (right) for different values of $D$. All other parameter values of Figs. are the same as Fig. 1.

In modelling the Gompertz and logistic growth laws driven by cross-correlated noises for the case of nonzero correlation time, we implement Eq. (9) instead of Eq. (8) in our probability distribution calculation (Eq. 13).

\section{A. Gompertzian growth}

First we study the effect of $\lambda$ on $p_{s t}(x)$ as function of $x$ for a fixed value of correlation time $\tau=0.3$. This is presented in Fig.6 $(a)$. This figure shows that $p_{s t}(x)$ as a function of $x$ decreases at smaller values of $x$ i.e, below $x$ around 7.5 and then increases as we increase $\lambda$. For the higher values of $x$ the curves of different $\lambda$ merge with each other and die out. On comparing these results with the results presented in Fig. 1a for Gompertz growth model in the case of zero correlation time, we observe that, as expected there is no change in qualitative nature of the curves and these results follow the similar trend as the previous ones. However, here we are interested 


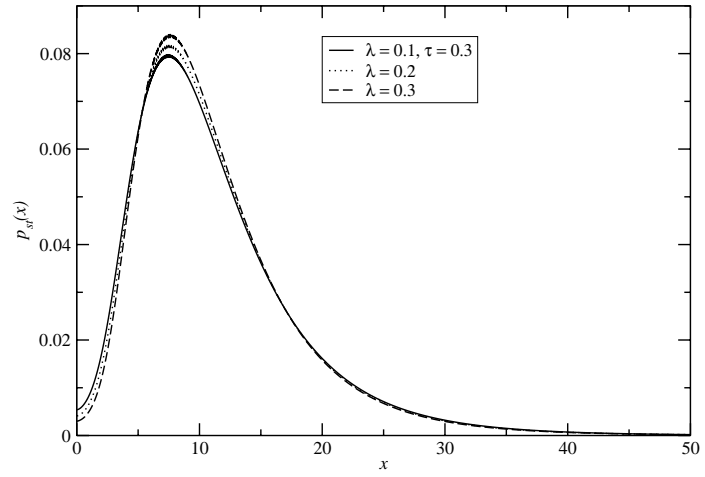

(a)

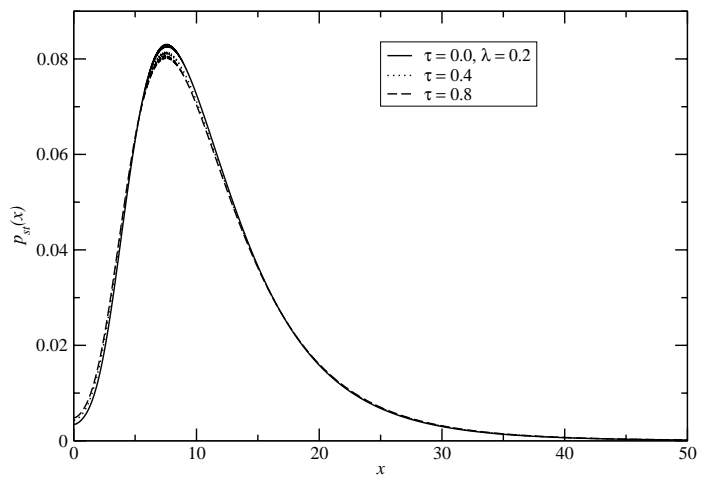

(b)

FIG. 6: (a) Plot of $p_{s t}(x)$ as a function of $x$ for different values of $\lambda$ at $\tau=0.3, D=0.3, \alpha=3.0, a=1$ and $b=0.1$. (b) Plot of $p_{s t}(x)$ as a function of $x$ for different values of $\tau$ at $\lambda=0.2$. All other parameter values are the same as $6(a)$. Parameter values are in arbitrary units.

in studying how both the correlation strength $\lambda$ and the correlation time $\tau$ affect the probability distribution. In Fig. 6b, we present the results of $p_{s t}(x)$ as a function of $x$ for a fixed value of correlation strength $\lambda=0.2$ and varying the correlation time $\tau$. As shown in the figure the probability as a function of $x$ decreases as we increase $\tau$. On comparing Figs. 6a and $6 \mathrm{~b}$, we find that the correlation strength $\lambda$ and the correlation time $\tau$ play the opposite role on the steady state probability i.e., $p_{s t}(x)$ increases with the increase of $\lambda$ and decreases with the increase of $\tau$.

To see the effect of $\lambda$ and $\tau$ on the cell population, we examine in Figs. $7 \mathrm{a}$ and $7 \mathrm{~b}$, the average cell population $\langle x\rangle$, as a function of $\tau$ for various values of $\lambda$ and as a function of $\lambda$ for different values of $\tau$, respectively. From these figures, we observe that $\langle x\rangle$ as a function of $\tau$ increases with increasing $\lambda$ and, viewed as a function of $\lambda$, decreases with increasing values of $\tau$. This also confirms that the correlation strength and the correlation time have the opposite effect on the average cell population in the case of Gompertz growth.

In Fig. 8 we examine the effect of $\tau$ on the average cell population $\langle x\rangle$ as a function of additive noise intensity $\alpha$ for

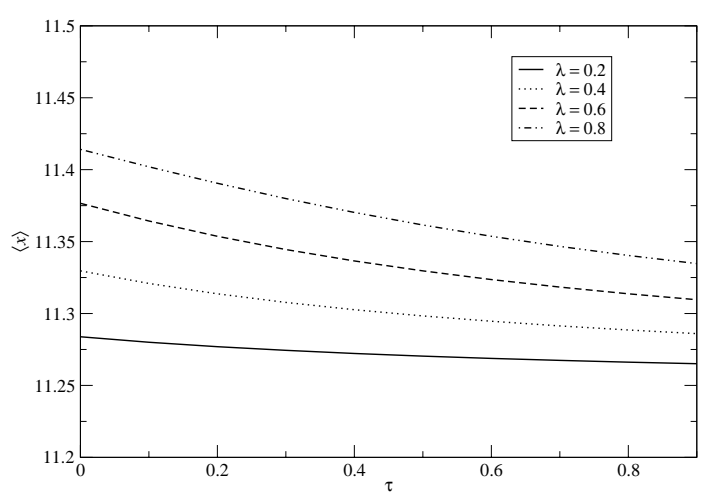

(a)

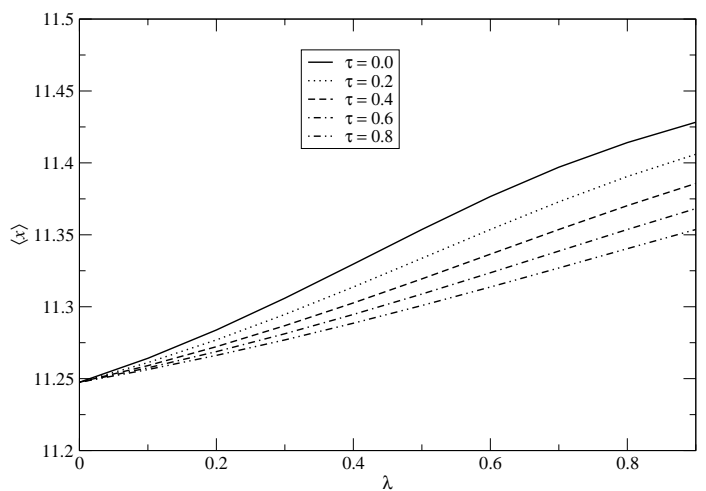

(b)

FIG. 7: Plot of $\langle x\rangle$ as a function of $\tau$ for different values of $\lambda$. (b) Plot of $\langle x\rangle$ as a function of $\lambda$ for different values of $\tau$. All other parameter values are the same as Fig. 6.

different values of multiplicative noise intensity $D$ at a fixed $\lambda=0.1$. $\langle x\rangle$ as a function of $\alpha$ decreases for some fixed values of $D$ and then increases showing a negative resonance-like characteristic and these resonance-like characteristics die out as $D$ is increased. However, there is no change in qualitative nature of the curve with the change in $\tau$, i.e., the correlation time has no effect on the resonance-like characteristic (see left and right figures, these are for different $\tau=0.2$ and 0.6). Similiar results have been obtained for the logistic models.

\section{B. Logistic growth}

We performed similar calculations for the nonzero correlation time for the logistic growth. As expected, since, logistic growth law predicts similiar behaviour to the Gompertz growth law, we obtained qualitatively similar behaviour of the curves in case of the logistic growth laws (see Figs. 7a and 7b). Similar calculations [10] have also been carried out in the case of logistic growth. However, they have shown that the correlation strength and the non-zero correlation time have the 


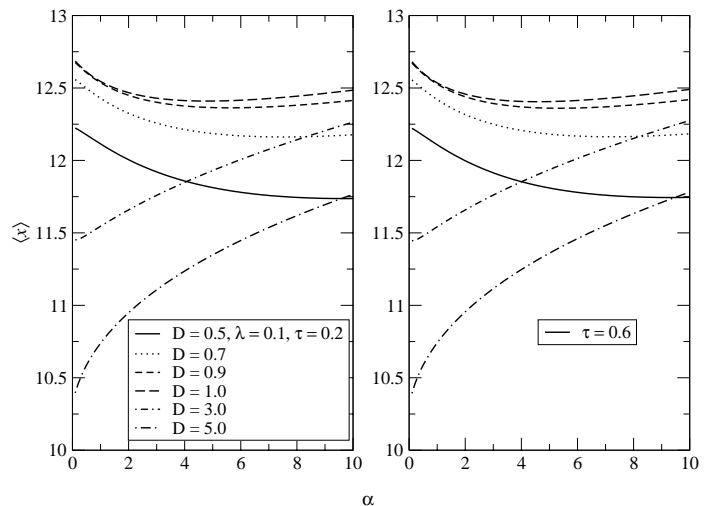

FIG. 8: Plot of $\langle x\rangle$ as a function of $\alpha$ for different values of $D$ at a fixed $\lambda=0.1$. Parameter values are the same in both the left and right hand figures except $\tau=0.2$ (left figure) and $\tau=0.6$ (right figure).

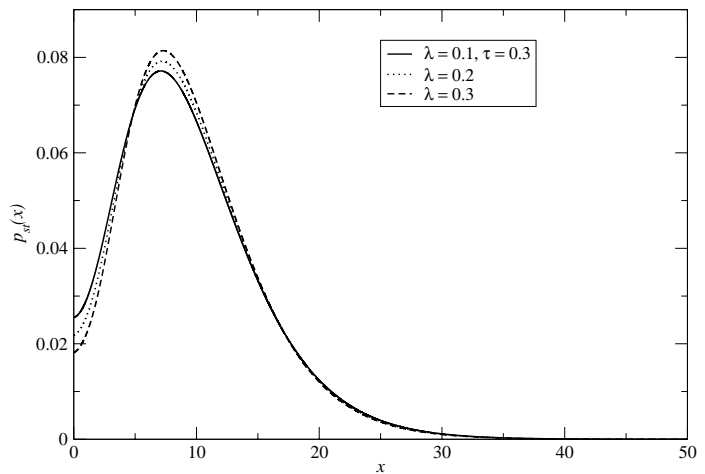

(a)

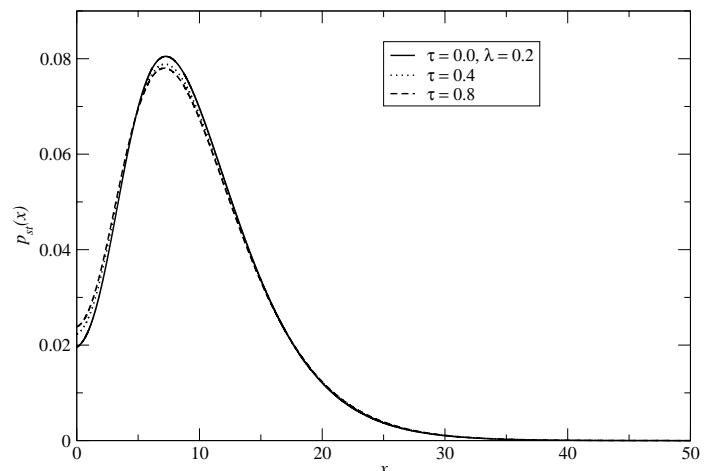

(b)

FIG. 9: Same as Fig. 6.

opposite effect on $p_{s t}(x)$ contrary to our findings in the present calculations. The discrepancy between our results and those of [10] in this section are reflected in the fact that [10] have based their model on the original model of [9].

We also studied the effect of nonzero correlation time on average cell population (Figs. 10a and 10b). We observe that

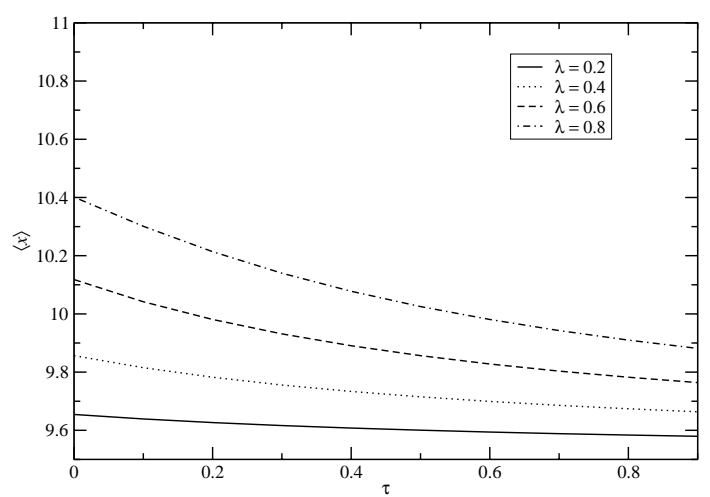

(a)

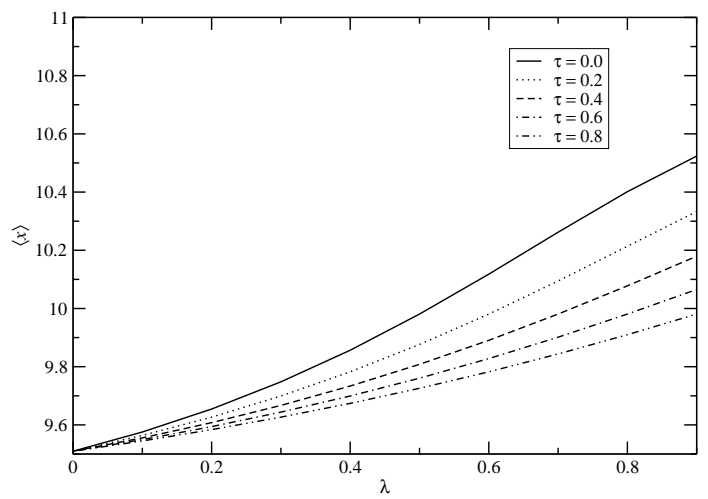

(b)

FIG. 10: Same as Fig. 7.
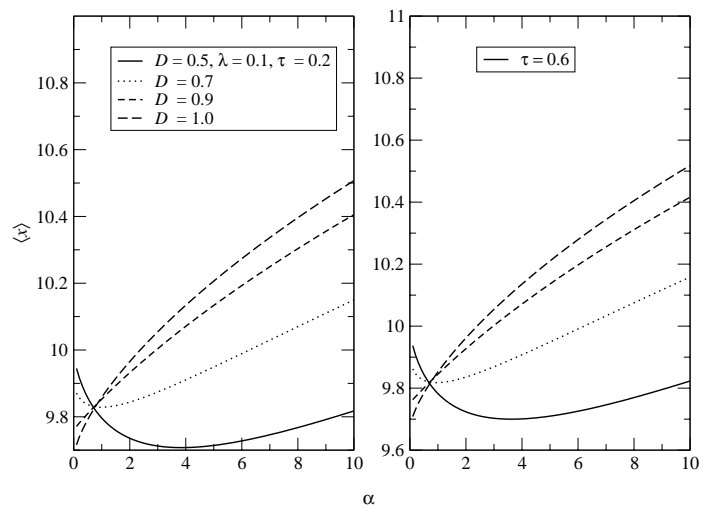

FIG. 11: Same as Fig. 8.

the correlation strength and the correlation time play opposite roles, which is similar to the case of the Gompertz growth law, in Sec. IV.A. We also find the average cell population increases with increasing $\lambda$ and decreases with increasing $\tau$. These results are also in qualitative disagreement with the calculations of [10] for reasons already outlined above. 
As in the Gompertz model, we study the effect of $\tau$ at a fixed $\lambda=0.1$ on the average cell population $\langle x\rangle$ as a functon of additive noise intensity $\alpha$ for different values of multiplicative noise intensity $D$ in the case of logistic growth model and the results are shown in Fig.11. As one can see these results are analogous to the results obtained in the case of zero correlation time in the logistic model and show similar resonancelike characteristics. However, as in the case of Gompertz growth law here we also observe that there is no effect of $\tau$ on the resonance.

\section{CONCLUSIONS}

We have considered the steady state properties of tumor cell growth, average cell populations and the effect of correlated noise using the Gompertz model. Our results were compared with existing calculations of the logistic model [9, 19] and [10]. In each of the Gompertz and logistic models we found that the both negative and positive correlation between additive and multiplicative noise were important in predicting the likely outcome of treatment protocols. From our results it is also observed that an inappropriate noise intensity can lead to the malignant growth of tumor cells. Another interesting result we found, is that multiplicative noise induces a phase transition and resonance in tumor growth. This behaviour arises due to the sigmoidal nature of both the Gompertz and logistic models which both have points of inflection that are always a fixed proportion of their asymptotic values. It would be interesting to pursue this study further, in order to understand the underlying mechanism(s) for the negative stochastic resonance like characteristic in the Gompertz and logistic non- bistable models.

We have studied the stationary properties of the Gompertz and logistic tumor cell growth models in the case of nonzero correlation time between additive and multiplicative noises. We found that the correlation time and the correlation strength play opposite roles on the steady state probability distribution in case of both the Gompertz and logistic model. As we increased the correlation strength, the steady state probability and the average cell population increased. This scenario is reversed if the correlation time is increased. Similarly to the case of nonzero correlation time, we observed a phase transition and resonance like characteristics in each of the Gompertz and logistic models. These phenomena are unaffected with the change of correlation time. It is worth mentioning here that in the logistic model we found our calculations significantly differed from those of both [9] and [10]. These results can be reproduced if one considers a negative correlation strength $(\lambda)$ between additive and multiplicative noise $[19,20]$. Finally there were no significant differences in the interpretation of the results between our simulations for the Gompertz and logistic models. This is probably due to the fact that these growth models belong to the same class of sigmoid function. These results thus provide strong evidence of a universal behaviour of sigmoid laws which are present in several problems of biological growth.

\section{Acknowledgements}

We thank Dr Sahoo for useful discussions. One of us A. Behera acknowledges financial assistance for an APG Studentship awarded by Queen's University, Belfast. SFC O'Rourke acknowledges current support from the Leverhulme Trust (Grant No. F/00203/K).
[1] L. Gammaitoni, P. Hanggi, P. Jung, and F. Marchesoni, Rev. Mod. Phys. 70, 223 (1998).

[2] Ya. Jia and Jia-rong Li, Phys. Rev. Lett. 78, 994 (1997).

[3] A. A. Zaikin, J. Kurths, and L. Schimansky-Geie, Phys. Rev. Lett. 85, 227 (2000).

[4] J. D. Murray, Mathematical Biology I. Springer: Berlin. (2002).

[5] M. Molski and J. Konarski, Phys. Rev. E. 68, 021916 (2003).

[6] S. C. Ferreira Jr., M. L. Matrins, and M. J. Vilela, Phy. Rev. E 67, 051914 (2003).

[7] A. Bru, S. Albertos, J. A. Lopez, I. Asenjo-Garcia, and Bru, Phys. Rev. Lett. 92, 238101 (2004).

[8] G. Albano and V. Giorno, J. Theo. Biol. 242, 329-336 (2006).

[9] B. Q. Ai, X. J. Wang, G. T. Liu, and L. G. Liu, Phys. Rev. E. 67, 022903 (2003).

[10] D. C. Mei, C. W. Xei, and L. Zhang, Eur. Phys. J. B. 41, 107 (2004).

[11] M. Marusic, Z. Bajzer, J. P. Freyer, and S. Vuk-Pavlovic, Cell. Prolif.27, 73 (1994).
[12] G. G. Steel, Basic Clinical Radiobiology. Arnold: third edition. (2002).

[13] M. A. A. Castro, F. Klamt, V. A.Grieneisen, I. Grivicih, and J. C. F. Moreira, Cell. Prolif. 36, 65 (2003).

[14] M. Gyllenberg and G. F. Webb, Growth, Dev. and Aging. 53, 25-33 (1989).

[15] A. R. Kansal, S. Torquato, E. A. Chiocca, and T. S. Deisboeck, J. Theor. Biol. 207, 431 (2000).

[16] F. Kozusko and Z. Bajzer, Math. Biosci. 185, 153 (2003).

[17] H. Risken, The Fokker-Planck Equation (Springer: Berlin), 96 $-99(1996)$

[18] C. W. Gardiner, Handbook of Stochastic Methods. (Spinger: Berlin), 108 - 125 (2003).

[19] A. Behera and S. F. C. O'Rourke, Phys. Rev. E. 77, 013901 (2008).

[20] B. Q. Ai, X. J. Wang, and Li. Q. Liu, Phys. Rev. E. 77, 013902 (2008). 